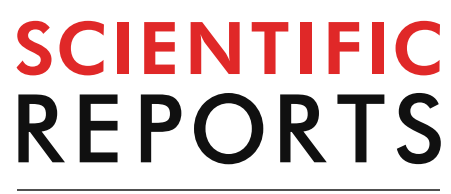

natureresearch

Check for updates

\title{
Prospective associations
} of the infant gut microbiome and microbial function with social behaviors related to autism at age 3 years

\author{
Hannah E. Laue ${ }^{1 \rrbracket}$, Susan A. Korrick ${ }^{2,3}$, Emily R. Baker ${ }^{4}$, Margaret R. Karagas ${ }^{1}$ \& \\ Juliette C. Madan ${ }^{1,5,6}$
}

The hypothesized link between gut bacteria and autism spectrum disorder (ASD) has been explored through animal models and human studies with microbiome assessment after ASD presentation. We aimed to prospectively characterize the association between the infant/toddler gut microbiome and ASD-related social behaviors at age 3 years. As part of an ongoing birth cohort gut bacterial diversity, structure, taxa, and function at 6 weeks $(n=166), 1$ year $(n=158)$, 2 years $(n=129)$, and 3 years $(n=140)$ were quantified with 16S rRNA gene and shotgun metagenomic sequencing $(n=101$ six weeks, $n=103$ one year). ASD-related social behavior was assessed at age 3 years using Social Responsiveness Scale (SRS-2) T-scores. Covariate-adjusted linear and permutation-based models were implemented. Microbiome structure at 1 year was associated with SRS-2 total T-scores $(p=0.01)$. Several taxa at 1, 2, and 3 years were associated with SRS-2 performance, including many in the Lachnospiraceae family. Higher relative abundance of Adlercreutzia equolifaciens and Ruminococcus torques at 1 year related to poorer SRS-2 performance. Two functional pathways, L-ornithine and vitamin $\mathrm{B} 6$ biosynthesis, were associated with better social skills at 3 years. Our results support potential associations between early-childhood gut microbiome and social behaviors. Future mechanistic studies are warranted to pinpoint sensitive targets for intervention.

Autism spectrum disorder (ASD), a combination of disordered social behaviors and repetitive or restricted interests, affects one in 34 boys and one in 145 girls in the United States, with altered behaviors presenting in some children as early as 18 months of age or younger ${ }^{1}$, but most children are not diagnosed until after age three ${ }^{2-8}$. Early symptoms of ASD include poor eye contact, problems with social behaviors (difficulty with joint attention, no response to their name, poor nonverbal communication), and poor imitation skills; many children are not evaluated until families identify a delay in verbal communication ${ }^{9}$. Diagnosing ASD as early as infancy and resultant early intervention has been shown to improve neurodevelopmental outcomes, highlighting the importance of identifying potential biomarkers for early detection ${ }^{10-12}$. Even among neurotypical children a spectrum of behaviors exists ${ }^{13}$, some of which may create challenges in personal relationships and learning and professional environments. While several risk factors for ASD have been identified or hypothesized including genetics $^{14}$, maternal infection during pregnancy ${ }^{15-17}$, and environmental exposures ${ }^{18}$, the etiologies are not well understood, leading to treatments targeting symptoms rather than underlying causes ${ }^{19}$. As a result, therapies, apart from early-life behavioral interventions, are often minimally effective $e^{20,21}$.

\footnotetext{
${ }^{1}$ Department of Epidemiology, Geisel School of Medicine at Dartmouth College, Hanover, NH, USA. ${ }^{2}$ Department of Environmental Health, Harvard T.H. Chan School of Public Health, Boston, MA, USA. ${ }^{3}$ Channing Division of Network Medicine, Department of Medicine, Brigham and Women's Hospital and Harvard Medical School, Boston, MA, USA. "Department of Obstetrics and Gynecology, Dartmouth-Hitchcock Medical Center, Lebanon, $\mathrm{NH}_{\text {, }}$ USA. ${ }^{5}$ Department of Pediatrics, Children's Hospital at Dartmouth, Lebanon, NH, USA. ${ }^{6}$ Department of Psychiatry, Children's Hospital at Dartmouth, Lebanon, NH, USA. ${ }^{\circledR}$ email: Hannah.E.Lave@Dartmouth.edu
} 
Recently, the interrelationship between the gastrointestinal tract and its microbiome (the compilation of microorganisms in the intestines) and the brain, known as the gut-brain axis, has been proposed as a potential cause or modifier of ASD behaviors ${ }^{22-24}$. Specifically, a recent mechanistic study found that mice transfected with the stool of ASD patients began displaying ASD behaviors, which were then modified by a correction of the imbalance in bacterial metabolites brought on by transfection ${ }^{25}$. Several case-control studies have compared the gut microbiome in children diagnosed with ASD to neurotypical siblings or community controls and observed notable differences ${ }^{26-33}$. However, these studies are subject to reverse causation, have been small, and have produced inconsistent results. To date, no epidemiological studies have examined the microbiome at multiple earlylife ages to elucidate when in the lifespan it is most relevant to neurodevelopment, particularly autism-related social behaviors. Further, no prospective cohort study has employed metagenomic sequencing and examined bacterial function in relation to social behaviors to better understand how gut bacteria may affect the brain.

Our study aimed to address these gaps by examining the infant/toddler microbiome at four early-life time points, beginning at 6 weeks postpartum, in relation to a continuous measure of social behavior at 3 years of age. In addition to investigating bacterial diversity, structure, and specific taxa, we inferred gene function from metagenomic sequences to identify pathways associated with essential features of ASD.

\section{Results}

Population characteristics. Study populations were similar across the four time points; most mothers reported not smoking during pregnancy $(>95 \%)$ and approximately half were parous (Table 1). Most parents were not of advanced age (average maternal age at delivery $32.3 \pm 4.6$ years, average paternal age at delivery $33.6 \pm 5.8$ years). SRS-2 total T-scores were lower (better) and had a narrower distribution than the normative population (a nationally representative sample of 474 ratings of 247 children $^{34}$ ) to which the test is standardized (i.e., approximate mean \pm SD: $44 \pm 5$ in each study population compared to $50 \pm 10$ in the normative sample). As expected, within subject diversity increased as the microbiome sample age increased [mean \pm SD Shannon Indices: $1.66 \pm 0.49,2.89 \pm 0.56,3.57 \pm 0.46$, and $3.83 \pm 0.48$ at 6 weeks, 1 year, 2 years, and 3 years, respectively; Table 1].

Within-subject diversity. At most time points, increased within-subject diversity was correlated with improved SRS-2 total T-scores $\left[\beta_{\text {Six-week Shannon }}=-0.19(-0.92,0.54), p=0.62 ; \beta_{\text {One-year Shannon }}=0.07(-0.60,0.74)\right.$, $p=0.83 ; \beta_{\text {Two-year Shannon }}=-0.04(-0.79,0.72), p=0.93 ; \beta_{\text {Three-year Shannon }}=-0.49(-1.25,0.27), p=0.21$ in basic models], although the relationships were not statistically significant (Fig. 1, Supplementary Table S1). Estimates were similar between basic and full models, but within-subject diversity at 6 weeks appeared to be more strongly correlated with SRS-2 total T-scores in the full model. Sensitivity analyses did not alter the conclusions (Supplementary Tables S2-S7). Using metagenomics data, all metrics of within-subject diversity at 1 year related to improved social behaviors. However, at 6 weeks higher Shannon and Simpson Indices were associated with better social behaviors, but higher taxa count related with worse social behavior (Supplementary Table S8). None of the estimates for metagenomic within-subject diversity reached statistical significance.

Microbial community structure. Community structure of the gut microbiome at 1 year was associated with total SRS-2T-scores at 3 years, particularly in the basic model $(p=0.01,0.06$ in basic and full models, respectively; Fig. 2, Supplementary Table S9). The $p$ values were similar between the basic and full models, except for 2-year community structure, where birth mode and peripartum antibiotic exposure explained significant portions of the variability in the full model (data not shown). Similar results were obtained in sensitivity analyses (Supplementary Table S10).

Individual taxa. The relative abundance of specific taxa at 1 year, 2 years, and 3 years were associated with social behaviors (Table 2). Higher relative abundance of two ASVs in the Lachnospiraceae (Blautia producta and an unknown taxon) at 1 year were associated with worse social behavior at 3 years [e.g., $\beta_{\text {B.producta-full }}=0.29$ $(0.15,0.42), q=0.017]$. Similarly, higher relative abundances of Coprococcus and Bifidobacterium at 2 years were associated with poorer social behaviors in both basic and full models. Two additional taxa (Ruminococcus gnavus and Sutterella) were associated with SRS-2 total T-scores in only the basic and full models, respectively [e.g., $\left.\beta_{\text {R.gnavus-basic }}=0.17(0.08,0.26), q=0.069\right]$. At 3 years, higher relative abundance of Butyricicoccus pullicaecorum was associated with worse social behavior in both basic and full models. In general, associations were modest, and the relative abundances of associated taxa were low (Table 2). Most of the statistically significantly associated taxa were in the Lachnospiraceae family (e.g., Blautia producta, Coprococcus), or more broadly in the Clostridiales order in the Firmicutes phylum (e.g., Butyricicoccus pullicaecorum), which was also true of the taxa with a nominal $p$ value $<0.05$ (Supplementary Table S11). Sensitivity and supplemental analysis results were similar (Supplementary Tables S12,S13).

Metagenomic data provided deeper insight into the taxa whose relative abundance at 6 weeks and 1 year were associated with SRS-2 total T-scores (Table 2). At 6 weeks, a taxon identified as Flavonifactor plautii was associated with worse total T-scores on the SRS-2. Similarly, at 1 year greater relative abundance of four taxa were associated with poorer social behavior, namely Adlercreutzia equolfaciens, Ruminococcus torques, Eubacterium dolichum, and a bacterium in the Lachnospiraceae, [e.g., $\beta_{\text {A.equolifaciens-full }}=0.002(0.001,0.002), q=0.001$ ]. Associations between taxa and SRS-2 total T-scores reaching a nominal $p$ value of 0.05 were largely in the Actinobacteria (class) and Clostridiales (Supplementary Table S14).

Bacterial functional pathways. Increased relative abundance of two functional pathways, L-ornithine de novo biosynthesis and the superpathway of pyridoxal 5'-phosphate biosynthesis and salvage, at both 6 weeks 


\begin{tabular}{|c|c|c|c|c|c|c|}
\hline & $\begin{array}{l}\text { NHBCS subjects } \\
\text { followed to age } 3 \text { years } \\
(\mathrm{n}=386)^{\mathrm{a}}\end{array}$ & $\begin{array}{l}\text { Six-week Cohort } \\
(\mathrm{n}=166)\end{array}$ & $\begin{array}{l}\text { One-year Cohort }{ }^{\mathrm{b}} \\
(\mathrm{n}=158)\end{array}$ & $\begin{array}{l}\text { Two-year Cohort } \\
(\mathbf{n}=129)\end{array}$ & $\begin{array}{l}\text { Three-year Cohort }{ }^{\mathrm{b}} \\
(\mathrm{n}=140)\end{array}$ & $\begin{array}{l}\text { Microbiome samples } \\
\text { at all ages }(n=21)\end{array}$ \\
\hline \multicolumn{7}{|c|}{ Parental characteristics } \\
\hline \multicolumn{7}{|l|}{ Parity } \\
\hline Nulliparous & $172(44.6)$ & $83(50)^{\mathrm{d}}$ & $77(49)$ & $64(50)$ & $61(44)$ & $12(57)$ \\
\hline Parous & $214(55.4)$ & $83(50)^{\mathrm{d}}$ & $81(51)$ & $65(50)$ & $79(56)$ & $9(43)$ \\
\hline \multicolumn{7}{|l|}{ Maternal education } \\
\hline College graduate or less & $251(65)$ & $93(56)$ & $92(58)$ & $79(61)$ & $86(61)$ & $12(57)$ \\
\hline $\begin{array}{l}\text { Any post-graduate } \\
\text { education }\end{array}$ & $135(35)$ & $73(44)$ & $66(42)$ & $50(39)$ & $54(39)$ & $9(43)$ \\
\hline \multicolumn{7}{|l|}{ Relationship status } \\
\hline Married & $355(92)$ & $156(94)$ & $150(95)$ & $120(93)$ & $132(94)$ & $19(91)$ \\
\hline $\begin{array}{l}\text { Never married or } \\
\text { separated }\end{array}$ & $31(8)$ & $10(6)$ & $8(5)$ & $9(7)$ & $8(6)$ & $2(9)$ \\
\hline \multicolumn{7}{|c|}{ Maternal smoking during pregnancy } \\
\hline No & $372(96.4)$ & $160(96)$ & $154(97)$ & $126(98)$ & $135(96)$ & $20(95)$ \\
\hline Yes & $14(3.6)$ & $6(4)$ & $4(3)$ & $3(2)$ & $5(4)$ & $1(5)$ \\
\hline Maternal age at delivery & $32.3( \pm 4.6)$ & $32.3( \pm 4.6)$ & $32.4( \pm 4.4)$ & $32.6( \pm 4.3)$ & $32.7( \pm 4.3)$ & $32.6( \pm 3.8)$ \\
\hline Paternal age at delivery & $33.6( \pm 5.8)$ & $33.6( \pm 5.9)$ & $33.5( \pm 5.7)$ & $33.5( \pm 5.4)$ & $34.1( \pm 5.7)$ & $34.1( \pm 3.9)$ \\
\hline \multicolumn{7}{|c|}{ Delivery characteristics } \\
\hline \multicolumn{7}{|l|}{ Delivery mode } \\
\hline Vaginal & $271(70.2)$ & $111(67)$ & $108(68)$ & $86(67)$ & $94(67)$ & $14(67)$ \\
\hline Caesarean & $115(29.8)$ & $55(33)$ & $50(32)$ & $43(33)$ & $46(33)$ & $7(33)$ \\
\hline \multicolumn{7}{|c|}{ Peripartum antibiotic exposure } \\
\hline Any & $176(45.6)$ & $94(57)^{d}$ & $81(51)^{\mathrm{d}}$ & $67(52)$ & $65(46)$ & $12(57)$ \\
\hline None & $210(54.4)$ & $72(43)^{d}$ & $77(49)^{\mathrm{d}}$ & $62(48)$ & $75(54)$ & $9(43)$ \\
\hline Gestational age (weeks) & $39.5( \pm 1.7)$ & $39.4( \pm 1.8)$ & $39.5( \pm 1.7)$ & $39.5( \pm 1.7)$ & $39.5( \pm 1.6)$ & $39.9( \pm 0.9)$ \\
\hline Birth weight $(\mathrm{g})$ & $3,424( \pm 539)$ & $3,396( \pm 546)$ & $3,458( \pm 569)$ & $3,470( \pm 572)$ & $3,445( \pm 503)$ & $3,494( \pm 391)$ \\
\hline \multicolumn{7}{|l|}{ Child characteristics } \\
\hline \multicolumn{7}{|l|}{ Feeding mode at 6 weeks } \\
\hline Exclusively breast fed & $206(53.4)$ & $90(54)$ & $84(53)$ & $71(55)$ & $79(56)$ & $10(48)$ \\
\hline $\begin{array}{l}\text { Formula fed or mixed } \\
\text { fed }\end{array}$ & $180(46.6)$ & $76(46)$ & $74(47)$ & $58(45)$ & $61(44)$ & $11(52)$ \\
\hline \multicolumn{7}{|l|}{ Infant sex } \\
\hline Male & $199(51.6)$ & $86(52)$ & $90(57)$ & $79(61)^{d}$ & $66(47)$ & $13(62)$ \\
\hline Female & $187(48.4)$ & $80(48)$ & $68(43)$ & $50(39)^{\mathrm{d}}$ & $74(53)$ & $8(38)$ \\
\hline $\begin{array}{l}\text { Age at SRS-2d follow-up } \\
\text { (years) }\end{array}$ & $3.1( \pm 0.2)$ & $3.1( \pm 0.3)$ & $3.1( \pm 0.3)$ & $3.1( \pm 0.3)$ & $3.1( \pm 0.2)$ & $3.1( \pm 0.3)$ \\
\hline $\begin{array}{l}\text { SRS-2 } 2^{\text {d total T-Score }} \\
\text { (unitless) }\end{array}$ & $43.7( \pm 4.7)$ & $43.5( \pm 4.6)$ & $43.2( \pm 4.5)$ & $43.6( \pm 4.4)$ & $43.9( \pm 4.5)$ & $43.3( \pm 4.3)$ \\
\hline \multicolumn{7}{|l|}{ Microbial diversity } \\
\hline $\begin{array}{l}\text { Shannon Index (unit- } \\
\text { less) }\end{array}$ & - & $1.66( \pm 0.49)$ & $2.89( \pm 0.56)$ & $3.57( \pm 0.46)$ & $3.83( \pm 0.48)$ & - \\
\hline $\begin{array}{l}\text { Simpson Index (unit- } \\
\text { less) }\end{array}$ & - & $0.68( \pm 0.16)$ & $0.88( \pm 0.09)$ & $0.94( \pm 0.04)$ & $0.95( \pm 0.03)$ & - \\
\hline Taxa count $(\#)$ & - & $33.1( \pm 11.8)$ & $90.1( \pm 35.1)$ & $166.2( \pm 45.1)$ & $208.2( \pm 67.6)$ & - \\
\hline
\end{tabular}

Table 1. Characteristics of New Hampshire Birth Cohort Study (NHBCS) subjects followed to age 3 years

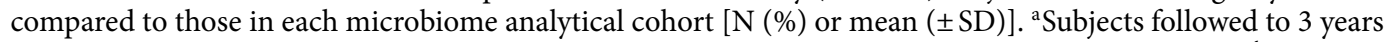
with complete covariate information ( 273 have microbiome sequenced in at least one stool sample). ${ }^{b}$ Overlap occurs between cohorts. ' Statistically different from complete cases not included in the analysis $(p<0.05)$. ${ }^{\mathrm{d}}$ Social Responsiveness Scale, 2nd edition.

and 1 year were associated with better social behavior at 3 years [e.g., $\beta_{\mathrm{L}-\text { ornithine-6W-basic }}=-2.4 \mathrm{E}-5(-4.2 \mathrm{E}-5$, $-6.8 \mathrm{E}-6), p=0.008$; Fig. 3, Supplementary Figure S1, Supplementary Table S15]. An additional three pathways (the superpathway of $\mathrm{L}$-aspartate and $\mathrm{L}$-asparagine synthesis, $\mathrm{O}$-antigen building blocks biosynthesis-Escherichia coli, and pentose phosphate pathway) had highly significant associations $(p<0.001)$ at 1 year in either basic or full models (Fig. 3, Supplementary Figure S1). Several associated pathways $(p<0.05)$ are involved in the urea cycle (involving aspartate, asparagine, or ornithine), pyridoxal 5'-phosphate (highly bioavailable vitamin B6) synthesis, or menaquinol biosynthesis (Supplementary Table S15). 


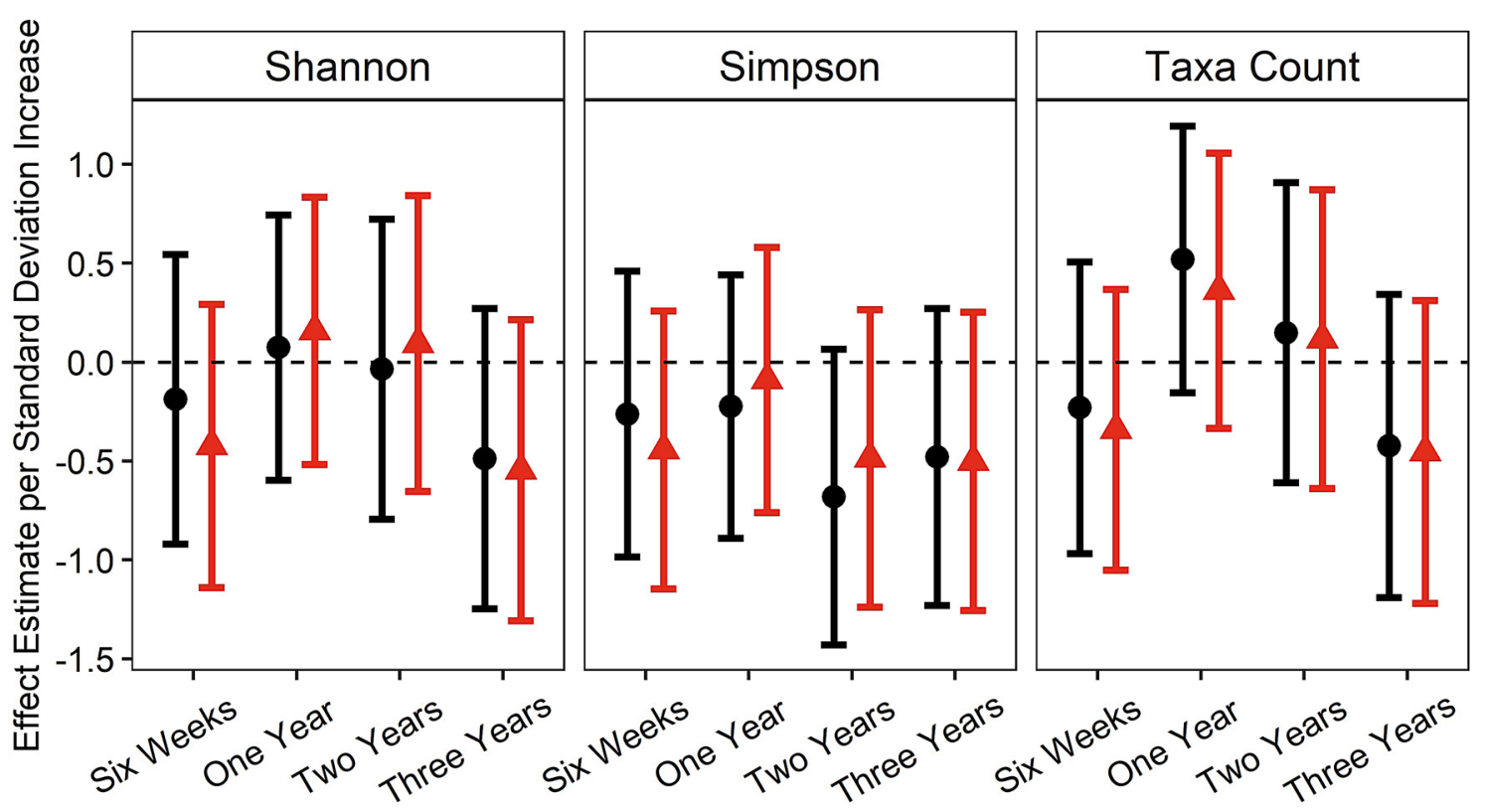

\section{Model - Basic $\&$ Full}

Figure 1. Associations of within-subject diversity with total T-scores on the Social Responsiveness Scale-2 (SRS-2) at 3 years of age. Basic models adjust for age at SRS-2, maternal education, marital status, maternal age, paternal age, child sex. Full models adjust for all covariates in the basic model and maternal self-reported smoking during pregnancy, early exclusive breastfeeding, delivery mode, peripartum antibiotics, and gestational age.

\section{Discussion}

Our analysis uncovered the potential for several novel, time-dependent associations between the early-life gut microbiome and child social behaviors at age three. Specifically, we found suggestive evidence of better social behaviors with increased within-subject microbial diversity during infancy and early childhood as well as an association with bacterial community structure at 1 year of life. Many of the taxa associated with relative decrements in social behaviors were in the Lachnospiraceae family. Metagenomic sequencing data from 6-week and 1-year fecal samples complemented our 16S rRNA findings and demonstrated several associations, including with Adlercreutzia equolifaciens and Ruminococcus torques. By classifying our metagenomic sequences into functional pathways, we also identified several promising pathways through which the microbiome may act on autism-related social behaviors.

Studies of within-subject diversity and health outcomes including attention deficit hyperactivity disorder ${ }^{35}$ and gastrointestinal disorders ${ }^{36}$, both common ASD comorbidities ${ }^{37,38}$, generally have found a beneficial association with increased microbial diversity. Our limited evidence of an association between within-subject diversity and social behaviors supports the potential for the microbiome to interact with the brain and behavior at a different level (e.g., the taxon level). The direction of the effect estimates in our study was somewhat sensitive to the metric used, with the Simpson Index (a measure of evenness) providing consistent evidence of potential beneficial impacts across time points and the Shannon Index (a measure of richness and evenness) and taxa count (a measure of richness) indicating a potential adverse association between increased diversity and social behaviors at 1 and 2 years $^{39}$. However, the lack of statistical significance implies our findings should be interpreted carefully.

The association with bacterial community structure was strongest when measured at 1 year, when the bacterial community is more established (as compared to 6 weeks) but more malleable than an adult microbiome ${ }^{40}$. However, because not all subjects appear in each analysis any time-specific effects may be artifacts of the specific population. Similar to the within-subject diversity analysis, the association between bacterial community structure and social behaviors did not appear to differ with the addition of autism risk factors for which community structure may act as a mediator.

Several taxa at 1,2, and 3 years were associated with poorer social behaviors, primarily in the Lachnospiraceae family, one of the most abundant in the gut. While the family was altered in several case-control investigations of ASD, the direction is not consistent across studies ${ }^{27-29}$, and is not present in others ${ }^{30,31}$. A prospective study examining the gut microbiome at six months and scores on the Ages and Stages Questionnaire (ASQ) at 3 years in the Vitamin D Antenatal Asthma Reduction Trial ( $\mathrm{n}=309$ primarily neurotypical children) found an adverse association between Ruminococcus, a genus in the Lachnospiraceae that was also identified in our analyses, and both total ASQ score and personal/social development ${ }^{41}$. We observed an association between a low-abundance Bifidobacterium taxon at two years and worse social behaviors. Bifidobacterium species are generally considered 


\section{Six Weeks}

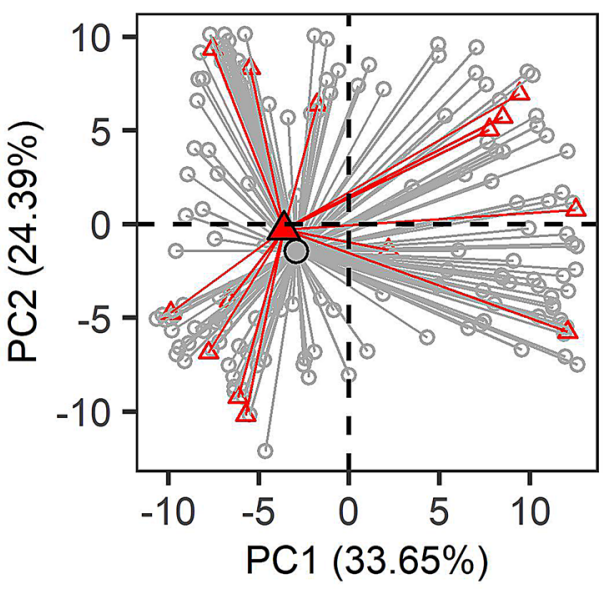

Two Years

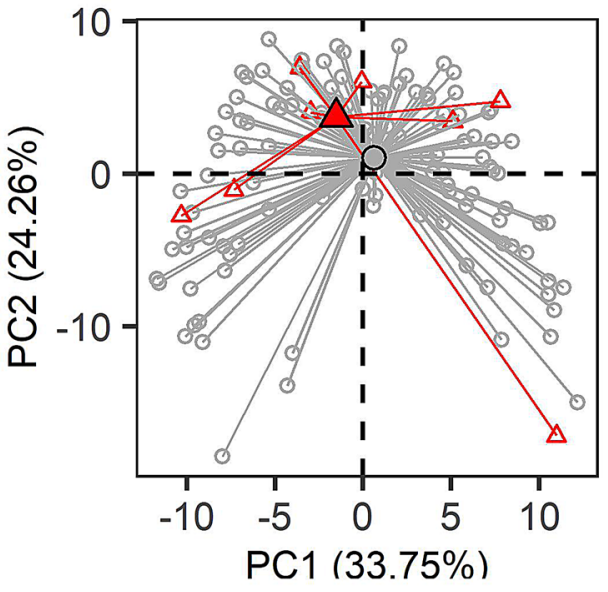

SRS2 Score
One Year

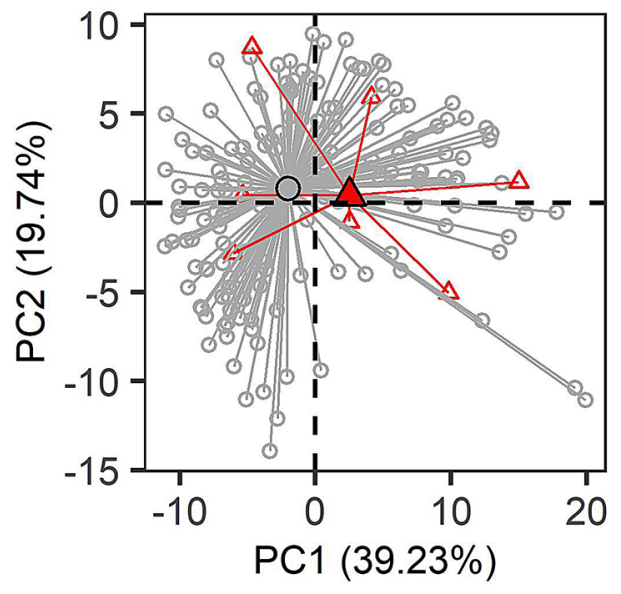

\section{Three Years}

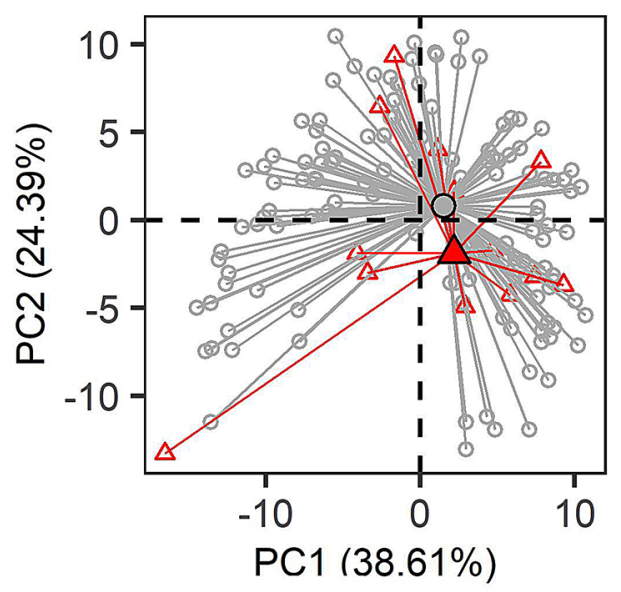

$<50$

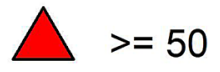

Figure 2. Generalized Unifrac distance principal component (PC) plots. Each point represents a subject and lines indicate distance to median centroids for subjects with Social Responsiveness Scale-2 (SRS-2) total T-scores above and below 50. Some points were removed for visual clarity ( 4 at one year and 1 at three years) but contributed to centroid calculation. Associations with continuous SRS-2 scores were statistically significant at 1 year ( $p=0.01,0.06$ in basic and full Models, respectively). Principal components are time point specific. The contribution of each principal component to overall variability in community structure is found along the axis (\%).

to be beneficial with respect to their psychoactive properties, especially in infants and children ${ }^{42-45}$. Because this finding was not consistent across time points or in our more probing shotgun metagenomics analyses it may be the result of chance. In our analysis using metagenomic data, which allows more precise identification of bacterial species and strain, we identified an adverse association between Ruminococcus torques, previously found to be increased in ASD cases with gastrointestinal symptoms $(n=54)$, and SRS-2 performance ${ }^{46}$. We also found an association between Adlercreutzia equolifaciens and poorer social behaviors. Although this taxon has not been detected in other ASD studies, its primary product, a phytoestrogen called equol, may interfere with normal microglial function ${ }^{47}$, including essential early-life synaptic pruning, a deficit of which is a putative mechanism for $\mathrm{ASD}^{48,49}$. Indeed, one recent study found increased genistein and daidzein (two other phytoestrogens dependent on gut microbial production) in mice colonized with ASD-patient microbiomes compared to controls ${ }^{25}$. Importantly, ASD phenotypes, including altered social behaviors, were reduced after restoring gut microbiome metabolomic balance.

In addition to more precisely identifying associations with select taxa, the use of metagenomic data allowed us to infer functional pathways the enrichment or depletion of which was associated with worse social behaviors. Two pathways were less abundant in children with better social behaviors in nearly all models: the L-ornithine de novo biosynthesis pathway and the superpathway of pyridoxal 5'-phosphate biosynthesis and salvage. In 


\begin{tabular}{|c|c|c|c|c|c|c|c|}
\hline Sequencing method & Microbiome age & Family & Genus & Species & $\begin{array}{l}\text { Estimate }(q) \text { in basic } \\
\text { model }^{\mathrm{a}}\end{array}$ & $\begin{array}{l}\text { Estimate }(q) \text { in full } \\
\text { model }^{\mathrm{a}}\end{array}$ & $\begin{array}{l}\text { Average relative } \\
\text { abundance (\%) }\end{array}$ \\
\hline \multirow{8}{*}{$16 \mathrm{~S}$ rRNA } & Six weeks & NA & & & & & \\
\hline & \multirow{2}{*}{ One year } & Lachnospiraceae & Blautia & producta & $0.34(0.01)$ & $0.29(0.017)$ & 0.1 \\
\hline & & Lachnospiraceae & & & $0.01(0.201)$ & $-^{c}$ & 0.01 \\
\hline & \multirow{4}{*}{ Two years } & Lachnospiraceae & Coprococcus & & $0.07(0.03)$ & $0.06(0.146)$ & 0.05 \\
\hline & & Lachnospiraceae & [Ruminococcus] & gnavus & $0.17(0.069)$ & $-^{c}$ & 0.2 \\
\hline & & Bifidobacteriaceae & Bifidobacterium & & $0.06(0.03)$ & $0.05(0.148)$ & 0.03 \\
\hline & & Alcaligenaceae & Sutterella & & $-^{c}$ & $0.01(0.211)$ & 0.01 \\
\hline & Three years & Ruminococcaceae & Butyricicoccus & pullicaecorum & $0.01(0.072)$ & $0.01(0.066)$ & 0.02 \\
\hline \multirow{5}{*}{ Metagenomics } & Six weeks & Ruminococcaceae & Flavonifactor & plautii & $0.002(0.052)$ & $-^{c}$ & 0.11 \\
\hline & \multirow{4}{*}{ One year } & Coriobacteriaceae & Adlercreutzia & equolifaciens & $0.002(0.004)$ & $0.002(0.001)$ & 0.07 \\
\hline & & Lachnospiraceae & [Ruminococcus] & torques & $0.01(0.01)$ & $0.01(0.042)$ & 1.13 \\
\hline & & Lachnospiraceae & & bacterium_6_1_63FAA & $0.001(0.01)$ & $0.001(0.067)$ & 0.04 \\
\hline & & Erysipelotrichaceae & Eubacterium & dolichum & $0.0003(0.038)$ & $-^{c}$ & 0.02 \\
\hline
\end{tabular}

Table 2. Taxa associated with Social Responsiveness Scale-2 (SRS-2) total T-scores at 3 years $(\mathrm{q}<0.25)$. ${ }^{a}$ MaAsLin models the microbiome as the outcome. Thus, estimate is increase in $\%$ relative abundance per point increase on SRS-2 total T-score. ${ }^{b}$ Mean relative abundance of specific amplicon sequence variant given microbiome age. ${ }^{c}$ Empty estimate cell indicates that the estimate was not significant $(q<0.25)$ in the given model.

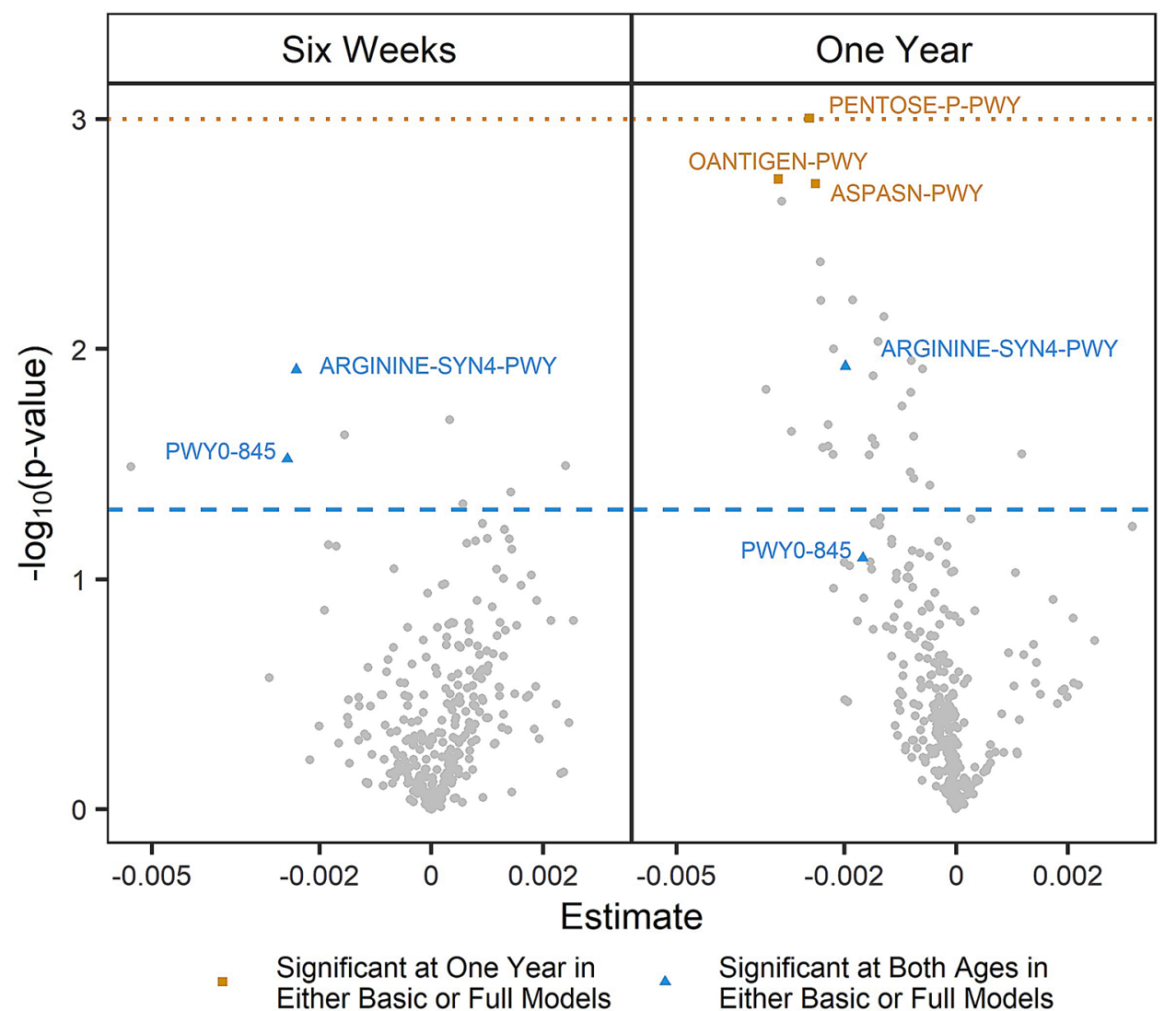

Figure 3. Associations of bacterial functional pathways and Social Responsiveness Scale-2 (SRS-2) total T-scores. Volcano plots of associations between metagenomically identified functional pathways and SRS-2 total T-scores in full models (adjusting for age at SRS-2, maternal education, marital status, maternal age, paternal age, child sex, maternal self-reported smoking during pregnancy, early exclusive breastfeeding, delivery mode, peripartum antibiotics, and gestational age). Points representing unmapped or unintegrated pathways have been removed for visual clarity ( 2 points per plot). Dashed blue line indicates $p=0.05$, dotted orange line indicates $p=0.001$. ARGININE-SYN4-PWY: L-ornithine de novo biosynthesis; ASPASN-PWY: superpathway of L-aspartate and L-asparagine synthesis; OANTINGEN-PWY: O-antigen building blocks biosynthesis (Escherichia coli); PENTOSE-P-PWY: pentose phosphate pathway; PWY0-845: superpathway of pyridoxal 5 '-phosphate biosynthesis and salvage. 
humans, ornithine is essential for the detoxification of ammonia, a neurotoxin, to urea ${ }^{50-52}$. Our findings are supported by a case-control study of ASD that found decreased urinary ornithine among cases provided that less excretion is an indication of increased salvage ${ }^{53}$. Although the authors of this study aspired to identify predictive biomarkers of ASD, their biospecimens, collected at an average of 5 years, were subject to reverse causation (i.e., autistic behaviors, with onset by 18 months $\mathrm{s}^{3-5}$, may impact diet or exposures, thus altering urinary amino acid concentrations). In contrast, our study indicates that as early as 6 weeks of life alterations in gut bacterial ornithine production are associated with social behaviors in childhood. The relative abundance of another amino acid synthesis pathway (superpathway of L-aspartate and L-asparagine synthesis) at 1 year was also associated with SRS-2 performance. Asparagine is essential to brain development and function ${ }^{54}$. Thus, further investigation of this pathway in ASD may be informative.

Our findings regarding the superpathway of pyridoxal 5'-phosphate (highly available vitamin B6) biosynthesis and salvage are relevant to clinical trials examining the effectiveness of high-dose vitamin B6 and magnesium supplementation in reducing autistic symptoms. While most of these studies have been small, and with conflicting results ${ }^{55-59}$, a more recent study suggests that a certain phenotypic subset of autistic subjects respond to B6 and magnesium supplementation ${ }^{60}$. Further research is needed to clarify whether/what gut microbial functions can identify responders so that treatment can be more successfully targeted. At 1 year we also found associations with $\mathrm{O}$-antigen building blocks biosynthesis and pentose phosphate pathway. $\mathrm{O}$-antigens are a vital component of lipopolysaccharides, which are hypothesized contributors to ASD, and demonstrated effectors of autism phenotypes ${ }^{61-63}$. The implications of the observed association with the pentose phosphate pathway, which is essential to glucose metabolism, are less clear. By generating nicotinamide adenine dinucleotide phosphate (NADPH) this pathway may contribute to the detoxification of reactive oxygen species, but this hypothesis must be robustly tested with in vitro models before any conclusion can be reached ${ }^{64}$.

Although this study suggests potential windows of microbial development that are sensitive for the development of social behaviors, our findings could have been population-specific rather than true effects because the populations comprise different individuals at each time point. In addition, despite our adjustment for numerous important confounders, we were not able to account for others including genetics. We chose not to include later antibiotic exposure in our models due to its lack of association with SRS-2 scores. However, this may have resulted in nondifferential exposure misclassification, adding imprecision to our estimates, but not biasing them. Further, due to limitations of statistical methods available for microbiome analysis (e.g., treating the microbiome as the dependent variable when it is the hypothesized exposure) and the constraints of observational data our findings cannot be interpreted as causal. However, as the first analysis to prospectively examine the microbiome at multiple time points early in life in relation to social development we have identified several important potential associations that should be explored further in mechanistic models.

The strengths of our study include careful adjustment for a range of variables that may confound the association between the microbiome and SRS-2 total T-scores or predict autism-like behavior. Further, by considering a model including variables for which the microbiome may act as a mediator we found that the association of the microbiome with social behaviors was largely independent of potential risk factors for autism (i.e., variables in the full model). Other variables-such as dietary factors and probiotics-were considered, but ultimately rejected, for model inclusion because of the likelihood of reverse causation (i.e., autism-related behaviors are known to affect food selectivity $)^{65}$. Ours is among one of the largest studies to date to consider the microbiome and autism-related behaviors, particularly in a prospective cohort setting. Our finding of differences as early as 6 weeks of life highlights opportunities for interventions that have been shown to change the direction of neurodevelopment in murine model ${ }^{25}$. Additionally, our findings in a pregnancy cohort are generalizable to nonclinical populations while still informing autism research. Specifically, because our cohort comprises primarily neurotypical children we expect stronger associations in a more neurodiverse population.

In summary, our findings suggest an association of the infant/toddler gut microbiome with social behaviors in a US population sample. Further research is warranted to elucidate mechanisms, and to determine whether features of the gut microbiome can be used to identify ASD during a vulnerable window during which time interventions can optimize neurodevelopmental outcomes.

\section{Methods and materials}

Study cohort. The New Hampshire Birth Cohort Study, an ongoing prospective pregnancy cohort, recruited pregnant women (ages 18-45) as previously described (Supplementary Methods) ${ }^{66}$. This analysis is based on a subset of children who provided a stool sample at 6 weeks, 1 year, 2 years, or 3 years and completed a social behavioral assessment at 3 years $(n=273$, Supplementary Figure S2, Supplementary Table S16). Although subjects overlapped in each of the populations, few $(n=21)$ had microbiome sequencing data at all four time points. Parents of participants provided written informed consent. Study protocols were reviewed and approved by the Center for the Protection of Human Subjects at Dartmouth, and all methods were carried out in accordance with relevant guidelines and regulations.

Stool sample collection and microbial sequencing. Stools were collected from infants/toddlers at 6 weeks, 1 year, 2 years, and 3 years postpartum. If the child was using diapers, the sample was collected by parents from a study-provided diaper, as previously described ${ }^{67}$. If the child was no longer using diapers, parents were provided an acid-washed receptacle that fit into the toilet to collect a urine-free stool sample (Supplementary Methods). Established protocols were followed for $16 \mathrm{~S}$ rRNA gene sequencing and data processing (Supplementary Methods) ${ }^{67-72}$. A subset of DNA samples (from 6-week and 1-year stools) also underwent metagenomic sequencing at Marine Biological Laboratory as previously described (Supplementary Methods) ${ }^{73-75}$. 
Assessment of social behaviors. Approximately 3 years postpartum, parents were asked to complete the Social Responsiveness Scale, 2nd edition, preschool form (SRS-2), which asks about their child's usual behavior $^{34,76}$. The SRS-2 is a standardized, validated instrument designed to assess social behavioral deficits and autistic traits as reflected in five components of social behavior (awareness, cognition, communication, motivation, and restricted interests/repetitive behavior) on a continuous scale ${ }^{77}$. SRS- 2 provides raw and agestandardized T-scores for each subscale as well as an overall total score. The T-scores are standardized to a mean (SD) of 50 (10) with higher scores indicating poorer social behavioral skills. For some visual representations of results scores were dichotomized at 50 points (approximately 1 SD above our population mean, 5-10\% of each study population above), but continuous total T-scores were used for all primary analyses. In clinical settings a more stringent cut off of 60 points is applied to identify individuals with mild social impairment.

Statistical analysis. Preliminary analyses identified factors associated with SRS-2 total T-scores in the study population and covariates were then selected if they were associated with SRS-2 scores (and in some cases the microbiome), but unlikely to be a factor for which the microbiome acts as a mediator. These included child age at follow-up, maternal education, maternal marital status, parity, maternal and paternal age at delivery, and child sex - the basic model. Additional models were run including autism risk factors for which the microbiome may act as a partial mediator (basic model variables and maternal smoking during pregnancy ${ }^{78}$, early postnatal exclusive breastfeeding ${ }^{79-81}$, delivery mode ${ }^{82}$, perinatal antibiotic exposure ${ }^{83}$, and continuous gestational age at delivery ${ }^{84}$ - the full model).

Shannon and Simpson Indices and taxa count were linearly regressed against SRS-2 total T-scores adjusting for covariates included in the basic and full models. The Shannon Index is reported as the primary within-subject diversity metric because it accounts for both richness and evenness of species ${ }^{85}$. Bacterial community structure was contrasted between subjects with generalized UniFrac (GUniFrac) distances ${ }^{86}$. To assess the significance of the relation between bacterial community structure and SRS-2 total T-scores we employed the adonis 2 function in the "vegan" package with 10,000 permutations ${ }^{87}$. Multivariate Association with Linear Models (MaAsLin2) was used to determine the association between individual amplicon sequence variants (ASVs) and total SRS-2Tscores $^{88,89}$. We restricted our analyses to ASVs with at least $0.001 \%$ relative abundance in at least $10 \%$ of subjects to reduce multiple testing. Any taxon-level association with an FDR-corrected q-value $<0.25$ was considered statistically significant, which is the MaAsLin default and is commonly used in microbiome studies where further investigation and validation of results is necessary ${ }^{90,91}$. A complementary analysis using diversities and taxon relative abundances derived from metagenomic data was conducted at 6 weeks and 1 year. Pathway relative abundances associated with SRS-2 total T-scores were determined with MaAsLin2, restricting to pathways with at least $0.0001 \%$ in at least $10 \%$ of samples. Due to the exploratory nature of this analysis, all pathways with a nominal $p$ value $<0.05$ were deemed of interest. Sensitivity analyses are described in Supplementary Methods.

\section{Data availability}

The 16S rRNA gene sequencing and shotgun metagenomic data used in this study are available through the National Center for Biotechnology Information (NCBI) Sequence Read Archive: https://ncbi.nlm.nih.gov/sra under accession number PRJNA296814.

Received: 29 May 2020; Accepted: 31 August 2020

Published online: 23 September 2020

\section{References}

1. CDC. Screening and Diagnosis|Autism Spectrum Disorder (ASD)|NCBDDD. Centers for Disease Control and Prevention https:// www.cdc.gov/ncbddd/autism/screening.html (2020).

2. Maenner, M. J. Prevalence of autism spectrum disorder among children aged 8 years-autism and developmental disabilities monitoring network, 11 sites, United States, 2016. MMWR Surveill. Summ. 69, 1-12 (2020).

3. Ozonoff, S., Heung, K., Byrd, R., Hansen, R. \& Hertz-Picciotto, I. The onset of autism: patterns of symptom emergence in the first years of life. Autism Res. 1, 320-328 (2008).

4. Johnson, C. P. \& Myers, S. M. Identification and evaluation of children with autism spectrum disorders. Pediatrics 120, 1183-1215 (2007).

5. Filipek, P. A. et al. Practice parameter: screening and diagnosis of autism: report of the Quality Standards Subcommittee of the American Academy of Neurology and the Child Neurology Society. Neurology 55, 468-479 (2000).

6. Barbaro, J. \& Dissanayake, C. Autism spectrum disorders in infancy and toddlerhood: a review of the evidence on early signs, early identification tools, and early diagnosis. J. Dev. Behav. Pediatr. 30, 447-459 (2009).

7. Shattuck, P. T. et al. The timing of identification among children with an autism spectrum disorder: findings from a populationbased surveillance study. J. Am. Acad. Child Adolesc. Psychiatry 48, 474-483 (2009).

8. Baio, J. Prevalence of autism spectrum disorder among children aged 8 years-autism and developmental disabilities monitoring network, 11 sites, United States, 2010. MMWR Surveill. Summ. 63, 1-24 (2014).

9. CDC. Data and Statistics on Autism Spectrum Disorder|CDC. Centers for Disease Control and Prevention https://www.cdc.gov/ ncbddd/autism/data.html (2020).

10. Dawson, G. et al. Randomized, controlled trial of an intervention for toddlers with autism: the Early Start Denver Model. Pediatrics 125, e17-23 (2010).

11. Mazurek, M. O., Curran, A., Burnette, C. \& Sohl, K. ECHO autism STAT: accelerating early access to autism diagnosis. J. Autism Dev. Disord. 49, 127-137 (2019).

12. Lai, M.-C., Lombardo, M. V. \& Baron-Cohen, S. Autism. The Lancet 383, 896-910 (2014).

13. Losh, M. et al. Neuropsychological profile of autism and the broad autism phenotype. Arch. Gen. Psychiatry 66, 518-526 (2009).

14. Chahrour, M. et al. Current perspectives in autism spectrum disorder: from genes to therapy. J. Neurosci. 36, 11402-11410 (2016).

15. Atladóttir, $\mathrm{H}$. Ó et al. Maternal infection requiring hospitalization during pregnancy and autism spectrum disorders. J. Autism Dev. Disord. 40, 1423-1430 (2010). 
16. Lee, B. K. et al. Maternal hospitalization with infection during pregnancy and risk of autism spectrum disorders. Brain Behav. Immun. 44, 100-105 (2015).

17. Jiang, H. et al. Maternal infection during pregnancy and risk of autism spectrum disorders: a systematic review and meta-analysis. Brain Behav. Immun. 58, 165-172 (2016).

18. Bölte, S., Girdler, S. \& Marschik, P. B. The contribution of environmental exposure to the etiology of autism spectrum disorder. Cell. Mol. Life Sci. 76, 1275-1297 (2019).

19. Masi, A., DeMayo, M. M., Glozier, N. \& Guastella, A. J. An overview of autism spectrum disorder, heterogeneity and treatment options. Neurosci. Bull. 33, 183-193 (2017).

20. McPheeters, M. L. et al. A systematic review of medical treatments for children with autism spectrum disorders. Pediatrics 127, e1312-1321 (2011).

21. Dawson, G. \& Burner, K. Behavioral interventions in children and adolescents with autism spectrum disorder: a review of recent findings. Curr. Opin. Pediatr. 23, 616-620 (2011).

22. Li, Q. \& Zhou, J.-M. The microbiota-gut-brain axis and its potential therapeutic role in autism spectrum disorder. Neuroscience 324, 131-139 (2016).

23. Carabotti, M., Scirocco, A., Maselli, M. A. \& Severi, C. The gut-brain axis: interactions between enteric microbiota, central and enteric nervous systems. Ann. Gastroenterol. 28, 203-209 (2015).

24. Kang, D.-W. et al. Long-term benefit of microbiota transfer therapy on autism symptoms and gut microbiota. Sci. Rep. 9, 1-9 (2019).

25. Sharon, G. et al. Human gut microbiota from autism spectrum disorder promote behavioral symptoms in mice. Cell 177, 16001618.e17 (2019).

26. Zhai, Q. et al. Disturbance of trace element and gut microbiota profiles as indicators of autism spectrum disorder: a pilot study of Chinese children. Environ. Res. 171, 501-509 (2019).

27. Liu, S. et al. Altered gut microbiota and short chain fatty acids in Chinese children with autism spectrum disorder. Sci. Rep. 9, 1-9 (2019).

28. Rose, D. R. et al. Differential immune responses and microbiota profiles in children with autism spectrum disorders and co-morbid gastrointestinal symptoms. Brain Behav. Immun. 70, 354-368 (2018).

29. Ma, B. et al. Altered gut microbiota in chinese children with autism spectrum disorders. Front. Cell. Infect. Microbiol. 9, 40 (2019).

30. De Angelis, M. et al. Fecal microbiota and metabolome of children with autism and pervasive developmental disorder not otherwise specified. PLoS ONE 8, e76993 (2013).

31. Strati, F. et al. New evidences on the altered gut microbiota in autism spectrum disorders. Microbiome 5, 24 (2017).

32. Wang, C., Geng, H., Liu, W. \& Zhang, G. Prenatal, perinatal, and postnatal factors associated with autism. Medicine (Baltimore) 96, e6696 (2017).

33. Kang, D.-W. et al. Reduced incidence of prevotella and other fermenters in intestinal microflora of autistic children. PLoS ONE 8 , e68322 (2013)

34. Bruni, T. P. Test review: social responsiveness scale-second edition (SRS-2). J. Psychoeduc. Assess. 32, 365-369 (2014).

35. Prehn-Kristensen, A. et al. Reduced microbiome alpha diversity in young patients with ADHD. PLoS ONE 13, e0200728 (2018).

36. Mosca, A., Leclerc, M. \& Hugot, J. P. Gut microbiota diversity and human diseases: should we reintroduce key predators in our ecosystem?. Front. Microbiol. 7, 455 (2016).

37. Simonoff, E. et al. Psychiatric disorders in children with autism spectrum disorders: prevalence, comorbidity, and associated factors in a population-derived sample. J. Am. Acad. Child Adolesc. Psychiatry 47, 921-929 (2008).

38. Kohane, I. S. et al. The co-morbidity burden of children and young adults with autism spectrum disorders. PLoS ONE 7, e33224 (2012).

39. DeJong, T. M. A comparison of three diversity indices based on their components of richness and evenness. Oikos 26, 222-227 (1975).

40. Yassour, M. et al. Natural history of the infant gut microbiome and impact of antibiotic treatments on strain-level diversity and stability. Sci. Transl. Med. 8, 343 ra81 (2016).

41. Sordillo, J. E. et al. Association of the infant gut microbiome with early childhood neurodevelopmental outcomes: an ancillary study to the VDAART Randomized Clinical Trial. JAMA Netw. Open 2, e190905-e190905 (2019).

42. O'Callaghan, A. \& van Sinderen, D. Bifidobacteria and their role as members of the human gut microbiota. Front. Microbiol. 7, 925 (2016).

43. Shaaban, S. Y. et al. The role of probiotics in children with autism spectrum disorder: a prospective, open-label study. Nutr. Neurosci. 21, 676-681 (2018).

44. Sarkar, A. et al. Psychobiotics and the manipulation of bacteria-gut-brain signals. Trends Neurosci. 39, 763-781 (2016).

45. Vuong, H. E. \& Hsiao, E. Y. Emerging roles for the gut microbiome in autism spectrum disorder. Biol. Psychiatr. 81, 411-423 (2017).

46. Wang, L. et al. Increased abundance of Sutterella spp. and Ruminococcus torques in feces of children with autism spectrum disorder. Mol. Autism 4, 42 (2013).

47. Villa, A., Vegeto, E., Poletti, A. \& Maggi, A. Estrogens, neuroinflammation, and neurodegeneration. Endocr. Rev. 37, 372-402 (2016).

48. Koyama, R. \& Ikegaya, Y. Microglia in the pathogenesis of autism spectrum disorders. Neurosci. Res. 100, 1-5 (2015).

49. Paolicelli, R. C. et al. Synaptic pruning by microglia is necessary for normal brain development. Science 333, 1456-1458 (2011).

50. Krebs, H. A. The discovery of the ornithine cycle of urea synthesis. Trends Biochem. Sci. 7, 76-78 (1982).

51. Wang, L. et al. Elevated fecal short chain fatty acid and ammonia concentrations in children with autism spectrum disorder. Dig. Dis. Sci. 57, 2096-2102 (2012).

52. Oja, S. S., Saransaari, P. \& Korpi, E. R. Neurotoxicity of ammonia. Neurochem. Res. 42, 713-720 (2017).

53. Liu, A. et al. Altered urinary amino acids in children with autism spectrum disorders. Front. Cell Neurosci. 13, 7 (2019).

54. Ruzzo, E. K. et al. Deficiency of asparagine synthetase causes congenital microcephaly and a progressive form of encephalopathy. Neuron 80, 429-441 (2013).

55. Findling, R. L. et al. High-dose pyridoxine and magnesium administration in children with autistic disorder: an absence of salutary effects in a double-blind placebo-controlled study. J. Autism Dev. Disord. 27, 467-478 (1997).

56. Mousain-Bosc, M. et al. Improvement of neurobehavioral disorders in children supplemented with magnesium-vitamin B6 II. Pervasive developmental disorder-autism. Magn. Res. 19, 53-62 (2006).

57. Rimland, B., Callaway, E. \& Dreyfus, P. The effect of high doses of vitamin B6 on autistic children: a double-blind crossover study. Am. J. Psychiatry 135, 472-475 (1978).

58. Kałużna-Czaplińska, J., Michalska, M. \& Rynkowski, J. Vitamin supplementation reduces the level of homocysteine in the urine of autistic children. Nutr. Res. 31, 318-321 (2011).

59. Nye, C. \& Brice, A. Combined vitamin B6-magnesium treatment in autism spectrum disorder. Cochrane Database Syst. Rev. 2005, CD03497 (2005)

60. Obara, T. et al. Potential identification of vitamin B6 responsiveness in autism spectrum disorder utilizing phenotype variables and machine learning methods. Sci. Rep. 8, 14840 (2018).

61. Fernández de Cossío, L., Guzmán, A., van der Veldt, S. \& Luheshi, G. N. Prenatal infection leads to ASD-like behavior and altered synaptic pruning in the mouse offspring. Brain Behav. Immun. 63, 88-98 (2017). 
62. Martin, C. R., Osadchiy, V., Kalani, A. \& Mayer, E. A. The brain-gut-microbiome axis. Cell Mol. Gastroenterol. Hepatol. 6, 133-148 (2018).

63. Srikantha, P. \& Mohajeri, M. H. The possible role of the microbiota-gut-brain-axis in autism spectrum disorder. Int. J. Mol. Sci. 20, 2115 (2019).

64. Fernandez-Marcos, P. J. \& Nóbrega-Pereira, S. NADPH: new oxygen for the ROS theory of aging. Oncotarget 7, 50814-50815 (2016).

65. Bandini, L. G. et al. Food selectivity in children with autism spectrum disorders and typically developing children. J. Pediatr. 157, 259-264 (2010).

66. Gilbert-Diamond, D. et al. Rice consumption contributes to arsenic exposure in US women. Proc. Natl. Acad. Sci. U. S. A. 108, 20656-20660 (2011).

67. Madan, J. C. et al. Association of cesarean delivery and formula supplementation with the intestinal microbiome of 6-week-old infants. JAMA Pediatr. 170, 212-219 (2016).

68. Caporaso, J. G. et al. Ultra-high-throughput microbial community analysis on the Illumina HiSeq and MiSeq platforms. ISME J. 6, 1621-1624 (2012).

69. Degnan, P. H. \& Ochman, H. Illumina-based analysis of microbial community diversity. ISME J. 6, 183-194 (2012).

70. Hoen, A. G. et al. Sex-specific associations of infants' gut microbiome with arsenic exposure in a US population. Sci. Rep. 8, 12627 (2018).

71. Callahan, B. J. et al. DADA2: high-resolution sample inference from Illumina amplicon data. Nat. Methods 13, 581-583 (2016).

72. DeSantis, T. Z. et al. Greengenes, a chimera-checked $16 \mathrm{~S}$ rRNA gene database and workbench compatible with ARB. Appl. Environ. Microbiol. 72, 5069-5072 (2006).

73. Coker, M. O. et al. Specific class of intrapartum antibiotics relates to maturation of the infant gut microbiota: a prospective cohort study. BJOG 127, 217-227 (2019).

74. Segata, N. et al. Metagenomic microbial community profiling using unique clade-specific marker genes. Nat. Methods 9, 811-814 (2012).

75. Franzosa, E. A. et al. Species-level functional profiling of metagenomes and metatranscriptomes. Nat. Methods 15, 962-968 (2018).

76. Constantino, J. N. \& Gruber, C. P. Social Responsiveness Scale-Second Edition (SRS-2) (Western Psychological Services, Los Angeles, 2012).

77. Bölte, S., Poustka, F. \& Constantino, J. N. Assessing autistic traits: cross-cultural validation of the social responsiveness scale (SRS). Autism Res 1, 354-363 (2008).

78. Levin, A. M. et al. Joint effects of pregnancy, sociocultural, and environmental factors on early life gut microbiome structure and diversity. Sci. Rep. 6, 31775 (2016).

79. Tseng, P.-T. et al. Maternal breastfeeding and autism spectrum disorder in children: a systematic review and meta-analysis. Nutr. Neurosci. 22, 354-362 (2019).

80. Boucher, O. et al. Association between breastfeeding duration and cognitive development, autistic traits and ADHD symptoms: a multicenter study in Spain. Pediatr. Res. 81, 434-442 (2017).

81. Julvez, J. et al. A cohort study on full breastfeeding and child neuropsychological development: the role of maternal social, psychological, and nutritional factors. Dev. Med. Child Neurol. 56, 148-156 (2014).

82. Yip, B. H. K. et al. Caesarean section and risk of autism across gestational age: a multi-national cohort study of 5 million births. Int. J. Epidemiol. 46, 429-439 (2017).

83. Hisle-Gorman, E. et al. Prenatal, perinatal, and neonatal risk factors of autism spectrum disorder. Pediatr. Res. 84, 190-198 (2018).

84. Lyall, K. et al. The changing epidemiology of autism spectrum disorders. Annu. Rev. Public Health 38, 81-102 (2017).

85. Shannon, C. \& Weaver, W. The Mathematical Theory of Communication (The University of Illinois Press, Champaign, 1948)

86. Chen, J. et al. Associating microbiome composition with environmental covariates using generalized UniFrac distances. Bioinformatics 28, 2106-2113 (2012).

87. Oksanen, J. et al. vegan: Community Ecology Package (2019).

88. Mallick, H. et al. Multivariable Association in Population-scale Metaomic Surveys (in submission).

89. Morgan, X. C. et al. Dysfunction of the intestinal microbiome in inflammatory bowel disease and treatment. Genome Biol 13, R79 (2012).

90. Lim, M. Y. et al. Analysis of the association between host genetics, smoking, and sputum microbiota in healthy humans. Sci. Rep. 6, 23745 (2016).

91. Leung, M. H. Y. et al. Changes of the human skin microbiota upon chronic exposure to polycyclic aromatic hydrocarbon pollutants. Microbiome 8, 100 (2020).

\section{Acknowledgements}

This study was supported by grants from the National Institute of Environmental Health (P01ES022832, P20ES018175, P42ES007373), the National Institute of General Medical Sciences (P20GM104416), the National Cancer Institute (R25CA134286) and the U.S. Environmental Protection Agency (RD-83544201, RD83459901).

\section{Author contributions}

H.E.L. conducted the formal analysis, drafted the initial manuscript including tables and figures, and reviewed and revised the manuscript. S.A.K., E.R.B., M.R.K., and J.C.M. conceptualized and designed the study, assisted with methodology, and reviewed and revised the manuscript. All authors approved the final manuscript as submitted and agree to be accountable for all aspects of the work.

\section{Competing interests}

The authors declare no competing interests.

\section{Additional information}

Supplementary information is available for this paper at https://doi.org/10.1038/s41598-020-72386-9.

Correspondence and requests for materials should be addressed to H.E.L.

Reprints and permissions information is available at www.nature.com/reprints.

Publisher's note Springer Nature remains neutral with regard to jurisdictional claims in published maps and institutional affiliations. 
(c) (i) Open Access This article is licensed under a Creative Commons Attribution 4.0 International cc) License, which permits use, sharing, adaptation, distribution and reproduction in any medium or format, as long as you give appropriate credit to the original author(s) and the source, provide a link to the Creative Commons licence, and indicate if changes were made. The images or other third party material in this article are included in the article's Creative Commons licence, unless indicated otherwise in a credit line to the material. If material is not included in the article's Creative Commons licence and your intended use is not permitted by statutory regulation or exceeds the permitted use, you will need to obtain permission directly from the copyright holder. To view a copy of this licence, visit http://creativecommons.org/licenses/by/4.0/.

(C) The Author(s) 2020 